\title{
New gas distribution system for the ATLAS RPCs
}

\author{
Giulio Aielli $^{\mathrm{a}, \mathrm{c}}$, Enrico Pastori ${ }^{\mathrm{b}, \mathrm{c},{ }^{*}}$ \\ ${ }^{a}$ University of Roma Tor Vergata \\ Via della Ricerca Scientifica 1, Roma, Italy \\ ${ }^{b}$ INFN Roma Tor Vergata \\ Via della Ricerca Scientifica 1, Roma, Italy \\ ${ }^{c}$ on behalf of the ATLAS MUON collaboration \\ E-mail: enrico.pastorieroma2.infn.it
}

\begin{abstract}
ATLAS RPCs is developed aimed at equalizing the flow with respect to the average counting rate per gas volume. The system takes into account the expected luminosity foreseen in superLHC (sLHC) and the consequent increase of gas flow necessary for the proper operation.
\end{abstract}

KEYWORDS: Gas systems and purification; Resistive-plate chambers; Large detector systems for particle and astroparticle physics;

\footnotetext{
${ }^{*}$ Corresponding author.
} 


\section{Contents}

1. Introduction 1

2. Gas flow equalization 2

3. Impedances for the gas distribution system 3

4. First check of the system and conclusions 5

\section{Introduction}

The Resistive Plate Chambers (RPCs) [1] detectors are used as muon trigger detectors in the ATLAS Experiment [2] at CERN-LHC accelerator. RPCs are gaseous detectors in which the quality of the gas mixture as well as the design of the gas supply system [3], play a fundamental role in their functioning.

The ATLAS RPC gas system consists of 2080 gas units with volumes ranging from 1.36 to 10.771 . The first parameter for the gas-flow equalization is the number of volume exchanges. This parameter has driven the project of the existing gas-distribution system (Fig.1) [4] that was planned to increase total flow ${ }^{1}$ with the increase of the ATLAS background. At the moment, as a consequence of some gas leaks, the gas-flow increase is practically impossible. Almost all of the gas-gap problems noticed until now are related to insufficient gas flow. We also realized that almost all of the found gas leaks are greater than expected due to a misbehaviour of the gas distribution at the existing gas-flow rate $^{2}$. For this reason and with a better understanding of the actual ATLAS background we considered the possibility to replace the old gas-distribution system.

The used gas mixture is
C4H10 (5\%) : SF6 $(0.3 \%)$

$>\quad$ The total gas flow is distributed by 5 gas racks

> 128 Input lines (manifolds)

$>128$ Output lines

$>$ Total Gas Volume of the whole detector: 13490.3

$>\quad$ Number of gas layers: 2080

(one or two gas-volume cascade connections)

$>\quad$ Number of Gas Volumes: 3592

$>$ Total Gas Flow: $5000 \mathrm{l} / \mathrm{h}$

$>\quad$ Now $\sim 0.3$ volume exchanges per hour 


\section{Gas flow equalization}

The average value of the measured current in each RPC gas volume (Fig.2), cleaned from all detector systematic contributions, represents the average charge per second delivered in the gas gap due to the incident particle rate. With the data collected in more than one year at a luminosity up to $3 \times 10^{33} \mathrm{~cm}^{-2} \mathrm{~s}^{-1}$ (about $1 / 3$ of the LHC design value), and with a better knowledge of the LHC-induced background [5], it is possible to choose a better gas equalization taking into account the measured charge per gas volume flowing through the detectors.

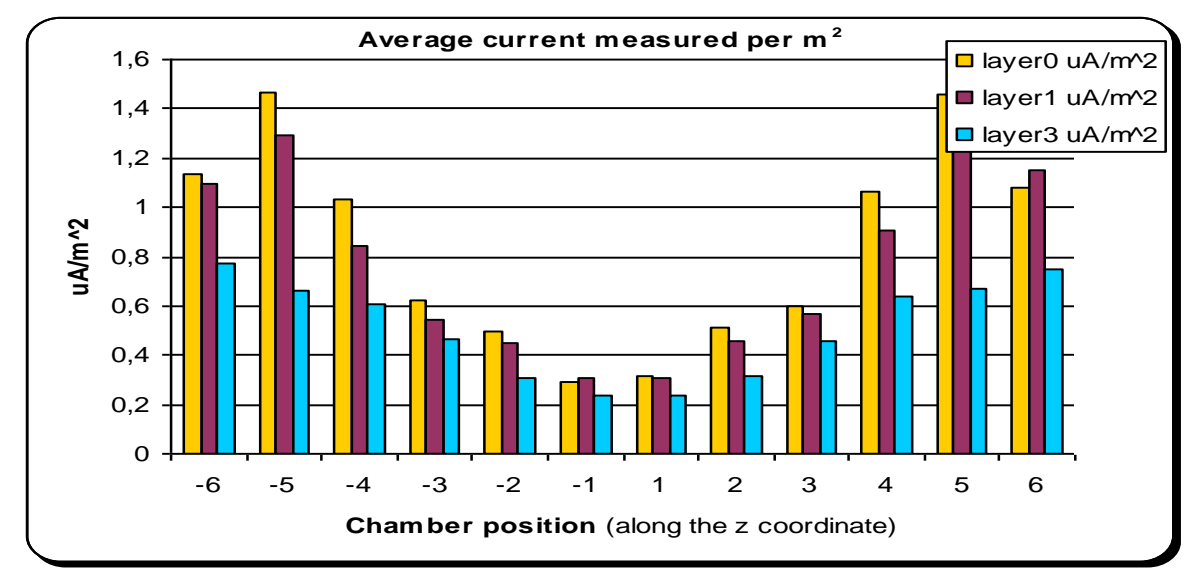

Figure 2: The average current per $\mathrm{m}^{2}$ measured. Notice the excess on chamber number 5 in both sides.

The flow needed in each RPC gas volume is proportional to the total charge rate delivered in the gas, which is in turn proportional to the electric current flowing through each RPC gap [6].

In any case as a precaution a minimum gas flow of 0.15 volume exchanges per hour has been granted to all the gas gaps irrespectively to the current, which corresponds to about one half of the total circulating flow.

Taking into account these two constraints, we have redistributed the total gas flow budget available to reduce the high tail of the deposited charge per unit volume distribution (Fig.4); this at the cost of providing a wider dynamic range through a completely new system of gas distribution (Fig.3). Indeed the charge deposited in the gas per unit volume inside the detectors is one of the most important parameters driving the RPCs to malfunctioning and faster ageing [7].

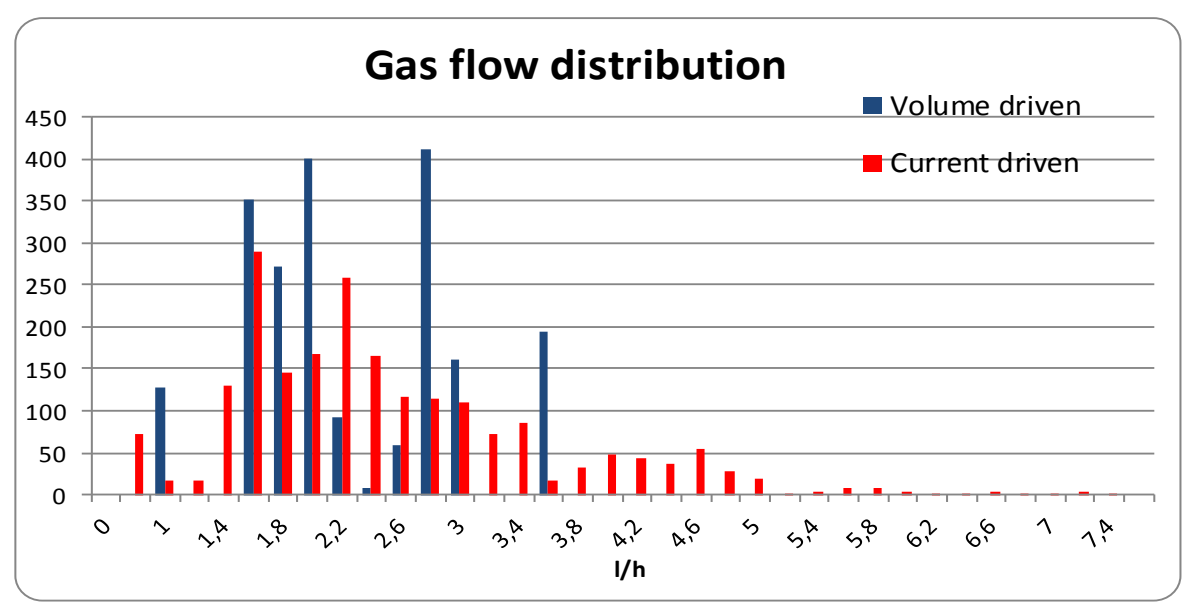

Figure 3: Gas flow distribution

$$
-2-
$$




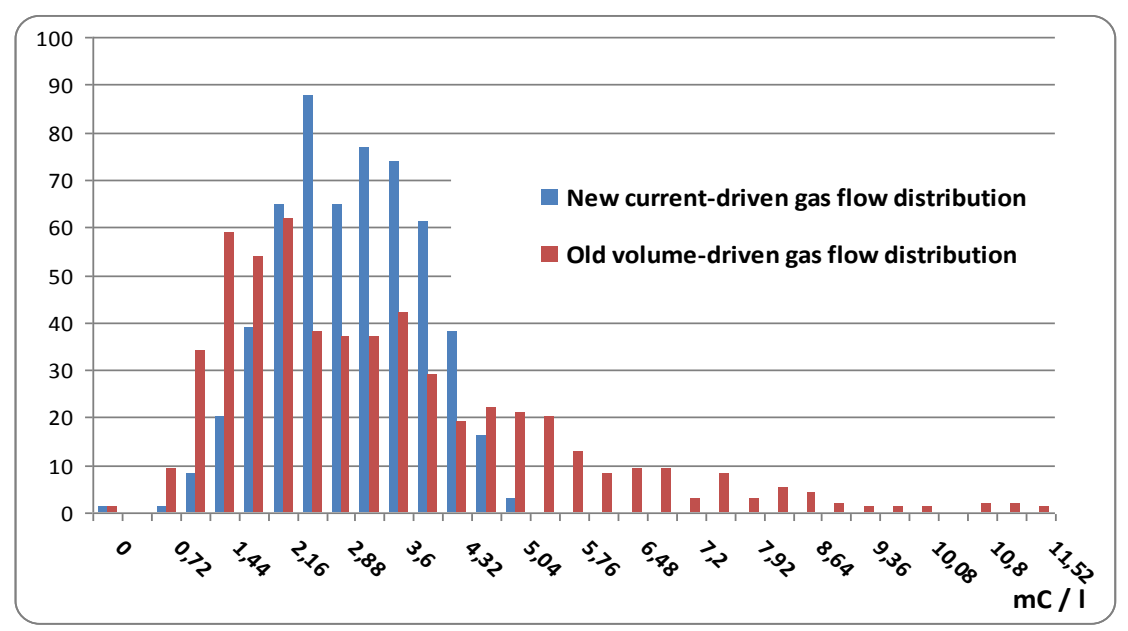

Figure 4: Charge deposited inside each detector per unit volume.

\section{Impedances for the gas distribution system}

The starting point in designing the system of distribution of the gas flow is the Hagen-Poiseuille law:

$$
\Delta P=\frac{8 \mu L}{\pi r^{4}} \Phi
$$

The equation (1) gives the pressure drop $(\Delta \mathrm{P})$ in a fluid with dynamic viscosity $\mu$ flowing through a cylindrical pipe of length $\mathrm{L}$ and radius $\mathrm{r}$ with the assumptions that the flow $\Phi$ is laminar, viscous and incompressible (in our case this is true because $\Delta \mathrm{P}<<$ atmospheric pressure) and the flow is through a hose of constant cross section and of a length substantially greater than its diameter. The flow of the fluid is turbulent for velocities above a threshold (Reynolds number greater than 2000), leading to larger pressure drops than those expected according to the Hagen-Poiseuille equation.

In the limit of validity of Hagen-Poiseuille law starting from a common manifold it is possible to distribute the gas in a certain flow in different gas layer by connecting a gas impedance of appropriate value in series with the assumption that the pressure drop in each gas layer is negligible compared to the pressure drop on the impedance.

We have set the pressure drop in each impedance to 6 mbar which is much greater than the pressure drop in each gas layer plus the return manifold pressure. The value of 6 mbar is situated well inside the range of values that can be set in the gas racks and is not so high as to become dangerous in the event of output block.

For the construction of these impedances, a set of commercial dosage needles (Tab.1) with a very strict tolerance of the inner diameter was chosen (the gas impedance depends on the fourth power of the inner diameter). In each needle the pressure drop at working point leads the gas velocity well below the turbulence point (Tab. 2).

\begin{tabular}{|c|c|c|c|c|c|c|c|c|c|c|c|c|c|c|c|}
\hline Needle Type & 1 & 2 & 5 & 6 & 7 & 8 & 9 & 10 & 11 & 12 & 13 & 15 & 16 & 17 & 18 \\
\hline $\mathrm{DI}(\mathrm{mm})$ & 254 & 254 & 305 & 330 & 330 & 330 & 330 & 406 & 406 & 406 & 406 & 508 &, 508 & 508 & 50 \\
\hline & 0 & 20 & 20 & 30 &, 20 & ,80 & 10 & 80 & , 20 & 80 & 3,10 & 30 & 20 & 0,80 & 3, \\
\hline & 0.802 & 083 & 676 & 9 & 0,332 & 0,342 & 0,486 & 0 , & 20 & 44 & 9 & 0,060 & 0,064 & 0,064 & 089 \\
\hline & 920 & 073 & 731 & 1,051 & 813 & 2,570 & 4,120 & 649 & 75 & 325 & 868 & 0,291 & 0,368 &, 610 & 735 \\
\hline
\end{tabular}

Table 1: Type of needles used. 


\begin{tabular}{|c|c|c|c|c|c|c|c|c|c|c|c|c|c|c|c|}
\hline \multirow[b]{2}{*}{ Gas Flow $\mathrm{l} / \mathrm{h}$} & \multicolumn{5}{|c|}{ Needle Type } & \multirow[b]{2}{*}{8} & \multirow[b]{2}{*}{9} & \multirow[b]{2}{*}{10} & \multirow[b]{2}{*}{11} & \multirow[b]{2}{*}{12} & \multirow[b]{2}{*}{13} & \multirow[b]{2}{*}{15} & \multirow[b]{2}{*}{16} & \multirow[b]{2}{*}{17} & \multirow[b]{2}{*}{18} \\
\hline & 1 & 2 & 5 & 6 & 7 & & & & & & & & & & \\
\hline 3 & 1289 & 1289 & 1074 & 992 & 992 & 992 & 992 & 807 & 807 & 807 & 807 & 645 & 645 & 645 & 645 \\
\hline 4 & 1719 & 1719 & 1432 & 1323 & 1323 & 1323 & 1323 & 1076 & 1076 & 1076 & 1076 & 860 & 860 & 860 & 860 \\
\hline 5 & 2149 & 2149 & 1790 & 1654 & 1654 & 1654 & 1654 & 1344 & 1344 & 1344 & 1344 & 1075 & 1075 & 1075 & 1075 \\
\hline 6 & 2579 & 2579 & 2148 & 1985 & 1985 & 1985 & 1985 & 1613 & 1613 & 1613 & 1613 & 1289 & 1289 & 1289 & 1289 \\
\hline 7 & 3009 & 3009 & 2506 & 2316 & 2316 & 2316 & 2316 & 1882 & 1882 & 1882 & 1882 & 1504 & 1504 & 1504 & 1504 \\
\hline 8 & 3439 & 3439 & 2864 & 2647 & 2647 & 2647 & 2647 & 2151 & 2151 & 2151 & 2151 & 1719 & 1719 & 1719 & 1719 \\
\hline 9 & 3868 & 3868 & 3221 & 2977 & 2977 & 2977 & 2977 & 2420 & 2420 & 2420 & 2420 & 1934 & 1934 & 1934 & 1934 \\
\hline 10 & 4298 & 4298 & 3579 & 3308 & 3308 & 3308 & 3308 & 2689 & 2689 & 2689 & 2689 & 2149 & 2149 & 2149 & 2149 \\
\hline 12 & 5158 & 5158 & 4295 & 3970 & 3970 & 3970 & 3970 & 3227 & 3227 & 3227 & 3227 & 2579 & 2579 & 2579 & 2579 \\
\hline 14 & 6017 & 6017 & 5011 & 4632 & 4632 & 4632 & 4632 & 3765 & 3765 & 3765 & 3765 & 3009 & 3009 & 3009 & 3009 \\
\hline 18 & 7737 & 7737 & 6443 & 5955 & 5955 & 5955 & 5955 & 4840 & 4840 & 4840 & 4840 & 3868 & 3868 & 3868 & 3868 \\
\hline 22 & 9456 & 9456 & 7875 & 7278 & 7278 & 7278 & 7278 & 5916 & 5916 & 5916 & 5916 & 4728 & 4728 & 4728 & 4728 \\
\hline 25 & 10745 & 10745 & 8949 & 8271 & 8271 & 8271 & 8271 & 6722 & 6722 & 6722 & 6722 & 5373 & 5373 & 5373 & 5373 \\
\hline 30 & 12894 & 12894 & 10738 & 9925 & 9925 & 9925 & 9925 & 8067 & 8067 & 8067 & 8067 & 6447 & 6447 & 6447 & 6447 \\
\hline
\end{tabular}

Table 2: Reynold numbers for each needle, leading to laminar flow (light green background), leading to turbulent flow (dark red background).

A sample of each type of needles has been tested measuring the pressure drop, with an accurate differential electronic manometer, at different gas flow rate, set with a well calibrated thermal mass flow controller. The test result (Fig.5) differs from the Hagen-Poiseuille law for the presence of a quadratic term. This term is due to the sharp change in the gas velocity, as it happens in a bent pipe or in an abrupt change of the pipe diameter where any slight perturbation could cause an excursion to turbulence.

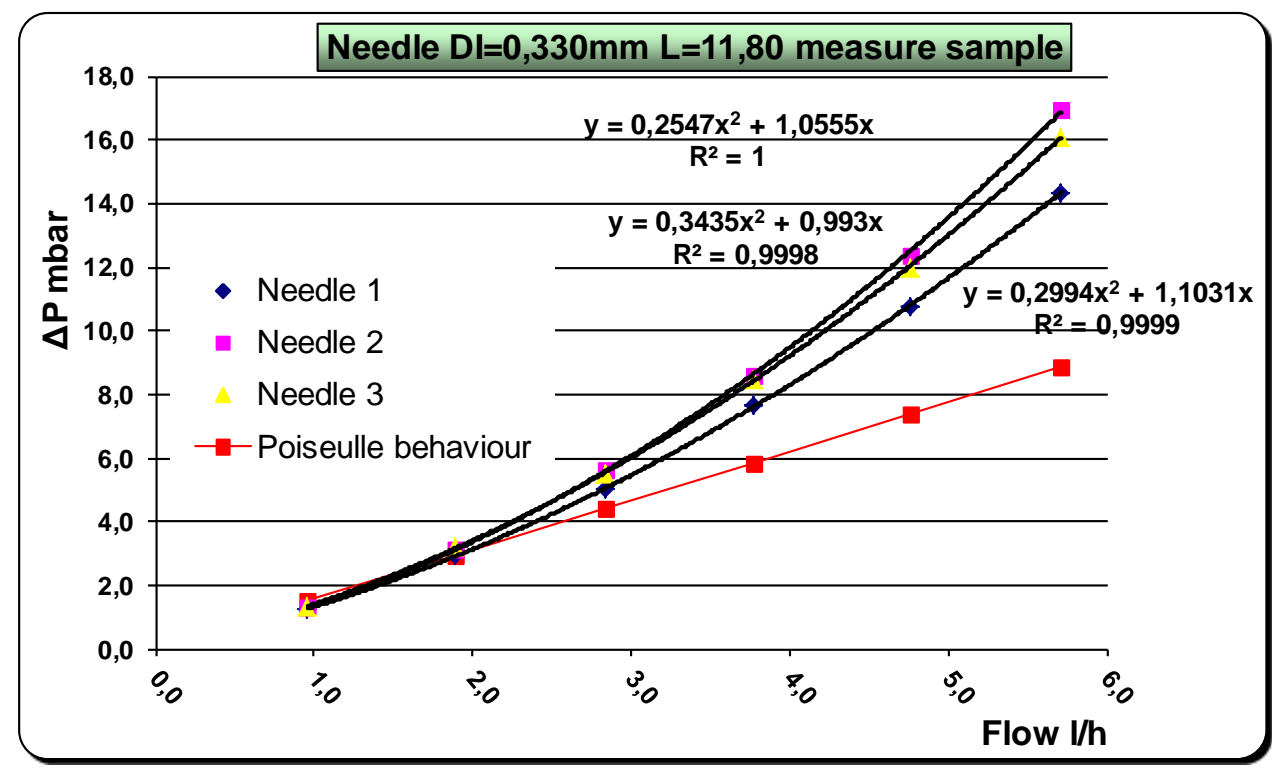

Figure 5: The measurements on a sample of needles of the same type. All measurements were done with pure TFE ( $\mu$ very close to the actual ATLAS gas mixture) and temperature in the range: $19^{\circ} \mathrm{C}-22^{\circ} \mathrm{C}$

The wide gas flow spread needed in the system has requested 18 different types of impedance (Tab. 3) built with one or two needles in series. 


\begin{tabular}{|c|c|c|c|c|c|c|c|c|c|c|}
\hline \multirow{2}{*}{$\begin{array}{c}\text { Impedance type } \\
1\end{array}$} & Needle 1 & Needle 2 & a1 & b1 & a2 & b2 & a-tot & b-tot & $\begin{array}{l}\text { Flow } \\
\text { I / h }\end{array}$ & Number needed \\
\hline & 1 & 2 & 0.80 & 2.92 & 1.08 & 4.07 & 1.89 & 6.99 & 0.72 & 72 \\
\hline 2 & 5 & 8 & 0.68 & 2.73 & 0.34 & 2.57 & 1.02 & 5.30 & 0.96 & 16 \\
\hline 3 & 5 & 7 & 0.68 & 2.73 & 0.33 & 1.81 & 1.01 & 4.54 & 1.07 & 12 \\
\hline 4 & 9 & & 0.49 & 4.12 & 0.00 & 0.00 & 0.49 & 4.12 & 1.27 & 40 \\
\hline 5 & 7 & 13 & 0.33 & 1.81 & 0.17 & 1.87 & 0.50 & 3.68 & 1.37 & 194 \\
\hline 6 & 5 & & 0.68 & 2.73 & 0.00 & 0.00 & 0.68 & 2.73 & 1.58 & 264 \\
\hline 7 & 8 & & 0.34 & 2.57 & 0.00 & 0.00 & 0.34 & 2.57 & 1.87 & 290 \\
\hline 8 & 6 & 11 & 0.30 & 1.05 & 0.12 & 0.78 & 0.42 & 1.83 & 2.19 & 252 \\
\hline 9 & 7 & & 0.33 & 1.81 & 0.00 & 0.00 & 0.33 & 1.81 & 2.32 & 160 \\
\hline 10 & 13 & & 0.17 & 1.87 & 0.00 & 0.00 & 0.17 & 1.87 & 2.60 & 202 \\
\hline 11 & 6 & & 0.30 & 1.05 & 0.00 & 0.00 & 0.30 & 1.05 & 3.05 & 158 \\
\hline 12 & 12 & & 0.16 & 1.33 & 0.00 & 0.00 & 0.16 & 1.33 & 3.23 & 100 \\
\hline 13 & 17 & 17 & 0.06 & 0.61 & 0.06 & 0.61 & 0.13 & 1.22 & 3.58 & 48 \\
\hline 14 & 16 & 17 & 0.06 & 0.37 & 0.06 & 0.61 & 0.13 & 0.98 & 4.03 & 110 \\
\hline 15 & 11 & & 0.12 & 0.78 & 0.00 & 0.00 & 0.12 & 0.78 & 4.54 & 100 \\
\hline 16 & 18 & & 0.09 & 0.73 & 0.00 & 0.00 & 0.09 & 0.73 & 5.07 & 30 \\
\hline 17 & 17 & & 0.06 & 0.61 & 0.00 & 0.00 & 0.06 & 0.61 & 6.03 & 22 \\
\hline 18 & 16 & & 0.06 & 0.37 & 0.00 & 0.00 & 0.06 & 0.37 & 7.24 & 10 \\
\hline & & & & & & & & & TOT & 2080 \\
\hline
\end{tabular}

Table 3: The 18 combinations of one or two needles in series used for assembling the 2080 impedances needed. For each impedance, the linear and quadratic term of both needles and the flow obtained with input pressure of 6 mbar are shown

Even doubling the pressure drop from 6 to 12 mbar the ratio of flow rates for each needle is on average a factor of 1.6 and not 2 as expected (Tab 4). This limit is almost insurmountable and is caused by the quadratic term in the relation between the pressure drop, $\Delta \mathrm{P}$, and gas flow, $\Phi$. To overcome this problem we are studying a different type of gas impedance made of many pipes assembled in a parallel configuration.

\begin{tabular}{|lc|c|c|c|c|c|c|c|c|c|c|c|c|c|c|} 
Needle Type & $\mathbf{1}$ & 2 & 5 & 6 & 7 & $\mathbf{8}$ & 9 & 10 & 11 & 12 & 13 & 15 & 16 & 17 & 18 \\
\hline Flow(6mbar) & 1,5 & 1,1 & 1,6 & 3,1 & 2,3 & 1,9 & 1,3 & 4,8 & 4,5 & 3,2 & 2,6 & 7,8 & 7,2 & 6,0 & 5,1 \\
\hline Flow(12mbar) & 2,5 & 1,9 & 2,7 & 4,8 & 3,9 & 3,3 & 2,3 & 7,5 & 7,3 & 5,4 & 4,6 & 11,9 & 11,1 & 9,7 & 8,2 \\
Flow(18mbar) & 3,3 & 2,6 & 3,5 & 6,2 & 5,1 & 4,4 & 3,2 & 9,6 & 9,4 & 7,2 & 6,2 & 15,0 & 14,2 & 12,7 & 10,7 \\
Flow(12mbar)/Flow(6mbar) & 1,7 & 1,7 & 1,7 & 1,6 & 1,7 & 1,7 & 1,8 & 1,6 & 1,6 & 1,7 & 1,7 & 1,5 & 1,5 & 1,6 & 1,6 \\
Flow(18mbar)/Flow(6mbar) & 2,2 & 2,3 & 2,2 & 2,0 & 2,2 & 2,4 & 2,5 & 2,0 & 2,1 & 2,2 & 2,4 & 1,9 & 2,0 & 2,1 & 2,1
\end{tabular}

Table 4: The flow driven for each needle at different starting-point pressure (6 mbar is the design pressure).

\section{First check of the system and conclusions}

After a test performed on detectors connected to one single gas rack the new gas impedances were installed in the entire gas system and have been working since the end of January 2012. The gas pressure in the racks inlet has increased to $7.5 \mathrm{mbar}$ (we expect 6 mbar on the new impedance and more than 1 mbar on the rack screw for line regulation). An accurate check was made on a large sample of chambers: the measured flow was compatible with the value of the installed impedance. With increasing luminosity of the LHC and the corresponding increase of the background, no problems have appeared in the chambers subject to lower irradiation where the gas flow was reduced. The chambers subject to higher irradiation are carefully monitored to ensure that the increase in gas flow in the new gas system is sufficient for a correct operation of the detectors.

We are confident that with this new gas distribution a good detector operation will be possible at the full-project sLHC luminosity without increasing the total gas flow more than a factor two. 


\section{References}

[1] R. Cardarelli and R. Santonico, Development of resistive plate counters, Nucl. Instrum. Meth. A 187 (1981) 377 ;

[2] ATLAS Collaboration, 2008 JINST 3 S08003

[3] De Asmundis, Application of Gas Chromatographic analysis to RPC detectors in the ATLAS experiment at CERN-LHC, J. Instrum. 2 (2007) T06001

[4] ATLAS Muon Collaboration, "Spectrometer Technical Design Report", CERN/LHCC/97-22, (1997);

[5] Aielli, G. Cavern background measurement with the ATLAS RPC system,PoS (RPC2012) 025

[6] Aielli, G. An RPC gamma irradiation test, Nucl.Instrum.Meth. A456 (2000) 82-86

[7] G. Aielli et al., Ageing test of the ATLAS RPCs at X5-GIF, Nucl. Instrum. Meth. A 533 (2004) 98. 